\title{
BMJ Open Protocol for a prospective, controlled, observational study to evaluate the influence of hypoxia on healthy volunteers and patients with inflammatory bowel disease: the Altitude IBD Study
}

Stephan Vavricka, ${ }^{1,2}$ Pedro A Ruiz, ${ }^{1}$ Sylvie Scharl, ${ }^{1}$ Luc Biedermann, ${ }^{1}$ Michael Scharl, ${ }^{1}$ Cheryl de Vallière, ${ }^{1,3}$ Carsten Lundby, ${ }^{3,4}$ Roland H Wenger, ${ }^{3,4}$ Leonhard Held, ${ }^{5}$ Tobias M Merz, ${ }^{6}$ Max Gassmann, ${ }^{3,7,8}$ Thomas Lutz, ${ }^{3,7}$ Andres Kunz, ${ }^{9}$ Denis Bron, ${ }^{9}$ Adriano Fontana, ${ }^{10}$ Laura Strauss, ${ }^{10}$ Achim Weber,, 11 Michael Fried, ${ }^{1}$ Gerhard Rogler, ${ }^{1,3}$ Jonas Zeitz ${ }^{1}$

To cite: Vavricka S, Ruiz PA, Scharl S, et al. Protocol for a prospective, controlled, observational study to evaluate the influence of hypoxia on healthy volunteers and patients with inflammatory bowel disease: the Altitude IBD Study. BMJ Open 2017;7:e013477. doi:10.1136/bmjopen-2016013477

- Prepublication history and additional material is available. To view please visit the journal (http://dx.doi.org/ 10.1136/bmjopen-2016013477).

Received 14 July 2016 Revised 25 November 2016 Accepted 2 December 2016

CrossMark

For numbered affiliations see end of article.

Correspondence to

Dr Jonas Zeitz;

jonas.zeitz@usz.ch

\section{ABSTRACT}

Introduction: Inflammatory bowel disease (IBD) is a chronic intestinal disorder, often leading to an impaired quality of life in affected patients. The importance of environmental factors in the pathogenesis of IBD, including their disease-modifying potential, is increasingly recognised. Hypoxia seems to be an important driver of inflammation, as has been reported by our group and others. The aim of the study is to evaluate if hypoxia can alter disease activity of IBD measured by Harvey-Bradshaw Activity Index in Crohn's disease (increase to $\geq 5$ points) and the partial Mayo Score for ulcerative colitis (increase to $\geq 2$ points). To test the effects of hypoxia under standardised conditions, we designed a prospective and controlled investigation in healthy controls and patients with IBD in stable remission.

Methods and analysis: This is a prospective, controlled and observational study. Participants undergo a 3-hour exposure to hypoxic conditions simulating an altitude of 4000 metres above sea level (m.a.s.l.) in a hypobaric pressure chamber. Clinical parameters, as well as blood and stool samples and biopsies from the sigmoid colon are collected at subsequent time points.

Ethics and dissemination: The study protocol was approved by the Ethics Committee of the Kanton Zurich (reference KEK-ZH-number 2013-0284). The results will be published in a peer-reviewed journal and shared with the worldwide medical community.

Trials registration number: NCT02849821;

Pre-results.

\section{INTRODUCTION}

Inflammatory bowel disease (IBD) is a chronic intestinal disorder, often leading to
Strengths and limitations of this study

- The prospective nature and the standardised study conditions are a clear strength of our study.

- A clinical study regarding the influence of hypoxia has never been performed on patients with inflammatory bowel disease before and we have a well-characterised data collection, including information on clinical status, and results on blood and sigmoidoscopy.

- A limitation is that psychological stress in the hypobaric chamber may lead to a release of pro-inflammatory cytokines that could influence inflammatory cascades.

- Another limitation is that a 3-hour exposure in the hypobaric chamber might be too short to show significant effects; however, if our analysis shows significant regulatory effects on inflammatory reactions, these would be even more marked at a longer exposure.

- A further limitation is that the sample size calculation is not based on the primary outcome of the study.

an impaired quality of life in affected patients. The aetiology of IBD is not fully understood, but the importance of environmental factors in the pathogenesis of this disease, including their disease-modifying potential, is increasingly recognised. As such, hypoxia seems to be an important driver of inflammation. ${ }^{1}{ }^{2}$ In healthy volunteers spending 3-4 days at an elevation of 3400 metres above sea level (m.a. s.l.), plasma levels of several inflammatory 
markers, such as interleukin (IL)-6, IL-1 receptor antagonist and $\mathrm{C}$ reactive protein, were found to be increased. ${ }^{3}$ Moreover, we recently reported on the association of IBD flare-ups and antecedent journeys to high-altitude regions and aircraft travels. Patients with IBD with at least one flare-up during a 12-month observation period were compared with a group of patients in remission. Patients with IBD experiencing flare-ups had more frequently undertaken flights and/or journeys to regions $>2000$ m.a. s.l. within 4 weeks of the flare-up when compared with patients in remission $(21 / 52(40.4 \%)$ vs $8 / 51$ (15.7\%), $\mathrm{p}=0.005){ }^{4}$

The role of hypoxia as an inductor of inflammation has also been studied extensively in vitro and in animal experiments. Several studies showed that in vitro and in vivo hypoxic conditions had an influence on the vascular barrier function, posthypoxic endothelial permeability and neutrophil tissue accumulation..$^{5-8}$ In addition, Rosenberger et a ${ }^{\ominus}$ analysed the role of hypoxia-inducible factor (HIF)-1 and hypoxia in inflammatory processes. They could show that HIF-1-dependent induction of netrin-1 attenuates hypoxiaelicited inflammation at mucosal surfaces.

Of note, hypoxia has been reported to be a diseasemodifying factor and pro-inflammatory stimulus in several diseases, such as IBD, rheumatoid arthritis and cancer. ${ }^{10-15}$ In addition, the impact of hypoxia on intestinal transport proteins, exercise capacity, sleep, nutrition, lung function, lipid metabolism and gastric emptying in healthy mountaineers has been investigated. It could be shown that, for example, reduced energy intake after rapid ascent to high altitudes was associated with severity of acute mountain sickness, but the underlying mechanisms are still unclear. Furthermore, intestinal transport was affected during systemic exposure to hypoxia in humans. ${ }^{16-18}$ Moreover, also iron uptake is controlled in an oxygen-dependent manner. ${ }^{19}$ Gastrointestinal problems are a well-known complication after high-altitude journeys. Anand $e t a t^{20}$ as well as $\mathrm{Wu}$ $e t a l^{21}$ showed that the exposure to high altitude may lead to gastroduodenal erosions and ulcer formation with consecutive gastrointestinal bleeding. In an own study, we investigated healthy volunteer mountaineers before and after an ascent to 4500 m.a.s.l. The participants underwent an endoscopy before the ascent and two further endoscopies at day 2 and day 4 after the ascent at $4500 \mathrm{~m}$. a.s.l. In the first baseline endoscopy, only one participant showed reflux oesophagitis, all other participants had a normal oesophagogastroduodenoscopy. At day 2 at 4500 m.a.s.l., $28 \%$ (4 out of 23) of the mountaineers showed pathological findings, including duodenal erosions/ulcers and haemorrhagic gastritis/duodenitis. At day $4,61 \%$ (16 out of 23 ) of the participants showed endoscopic abnormalities. These results underline that exposure to hypoxic conditions may have profound effects on the gastrointestinal tract. ${ }^{22}$

To evaluate the potential influence of hypoxia on the course of IBD on a cellular level and to analyse the effects of hypoxia under standardised conditions, we designed a prospective and controlled investigation in patients with IBD in stable remission and compared them with healthy volunteers. Our primary aim is to show that a 3-hour stay at high altitude might alter disease activity of IBD. A total of 10 healthy volunteers, 11 patients with Crohn's disease $(\mathrm{CD})$ and 9 patients with ulcerative colitis (UC) undergo a 3-hour exposure to hypoxic conditions simulating an altitude of 4000 m.a.s.l. $(13000 \mathrm{ft}$ Flight Level (FL)) in a hypobaric low-pressure chamber situated at the Swiss Aeromedical Center, Dubendorf, Switzerland. The hypobaric pressure chamber is set to pressure of $462 \mathrm{~mm} \mathrm{Hg}$ to simulate the conditions at 4000 m.a.s.l., the $\mathrm{PaO}_{2}$ is $7 \mathrm{kPa}$. Of note, the cabin pressure in a standard aeroplane correlates with 2000 to 2500 m.a.s.l., at 2000 m.a.s.l. the $\mathrm{PaO}_{2}$ is $10 \mathrm{kPa}$. Stool samples for the analysis of calprotectin and microbiota composition, biopsy samples from the rectosigmoid region and blood samples are repetitively collected and analysed in conjunction with detailed records of clinical symptoms over a subsequent interval of 4 weeks.

\section{METHODS AND ANALYSIS}

\section{Study design}

The study was designed as a prospective, controlled and observational study.

\section{Inclusion and exclusion criteria}

Patients are eligible if they

- Are $\geq 18$ and $<60$ years of age;

- Have a diagnosis of CD or UC at least 6 months prior to inclusion;

- Give written consent;

- Are in clinical remission (measured by HarveyBradshaw Activity Index (HBI) in CD and the partial Mayo Score for UC).

Patients were not eligible if they

- Have contraindications for a sigmoidoscopy;

- Have intercurrent bacterial or viral intestinal disease (by culture or serology);

- Are pregnant or breast feeding;

- Have a severe concomitant disease which excluded them from participating in the study by means of the study physician;

- Are likely to or showed no cooperation for the study procedures;

- Have active infection or systemic antibiotic, antiviral or antifungal treatment 3 weeks before baseline;

- Are suffering from short-bowel syndrome;

- Are receiving parenteral nutrition;

- Have a clinical condition which did not allow a stay at heights of 4000 m.a.s.l.;

- Are claustrophobic.

\section{Primary and secondary outcomes}

Primary outcome

A 3-hour stay at high altitude $(4000 \mathrm{~m}, 13000 \mathrm{ft}$ Flight Level (FL)) can alter disease activity of IBD measured by 
HBI in CD (increase to $\geq 5$ points) and the partial Mayo Score for UC (increase to $\geq 2$ points).

\section{Secondary outcomes}

- Change of the volume state of the body due to hypoxia (repetitive measurements of bladder volume; measurement of angiotensin and vasopressin in urine and measurement of renin, aldosterone, vasopressin and angiotensin in blood).

- Show that a 3-hour stay at high altitude $(4000 \mathrm{~m}$, $13000 \mathrm{ft}$ Flight Level (FL)) modulates biochemical pro-inflammatory response pathways in patients with IBD and healthy individuals, and investigate whether this response differs between the two groups.

\section{Procedure of the study}

In brief, patients undergo a 3-hour exposure to hypoxic conditions simulating an altitude of 4000 m.a.s.l. in a hypobaric low-pressure chamber situated at the Swiss Aeromedical Center, Dubendorf, Switzerland (figures 1 and 2). The pressure in the hypobaric low-pressure chamber was $462 \mathrm{~mm} \mathrm{Hg}$ and $\mathrm{PaO}_{2}$ was $7 \mathrm{kPa}$. Stool samples to analyse calprotectin and microbial composition, biopsy samples from the rectosigmoid region and blood samples are repetitively collected and analysed over a period of 4 weeks in conjunction with detailed records of clinical symptoms.

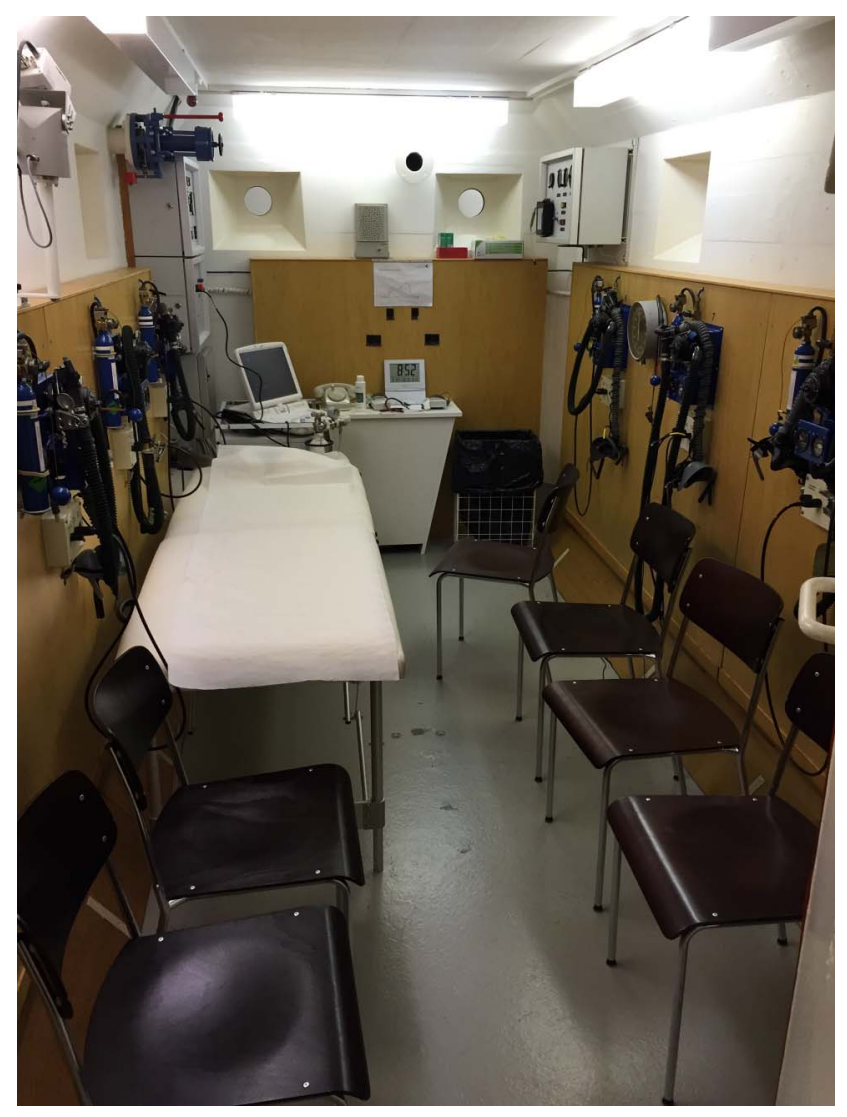

Figure 1 Hypobaric low-pressure chamber at the Swiss Aeromedical Center, Dubendorf, Switzerland.

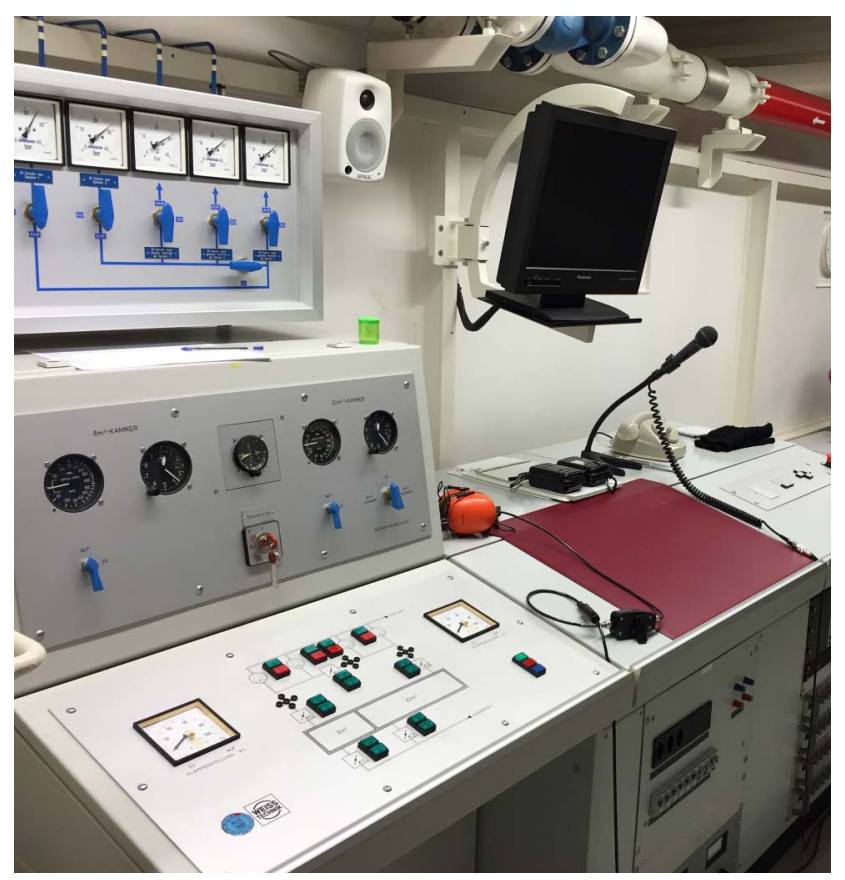

Figure 2 Control room of the hypobaric low-pressure chamber at the Swiss Aeromedical Center, Dubendorf, Switzerland.

In detail, the study is divided into screening (S), baseline (T0), visit 1 (T1), visit 2 (T2) and visit 3 (T3) (figure 3):

\section{Screening}

During the screening procedure, the inclusion and exclusion criteria are assessed, and the patients give written consent. The patients receive a clinical examination including an otolaryngological examination, as well as vital signs (heart rate and blood pressure) and body temperature assessment. Demographic data, personal medical history, medication and anamnesis are also assessed.

\section{Baseline (T0)}

Baseline (T0) takes place 1 day before the stay in the hypobaric chamber. At T0, inclusion and exclusion criteria are reassessed, patients receive a clinical examination; vital signs (heart rate and blood pressure), body temperature, medication, adverse events and HBI (patients with CD) or partial Mayo Score (patients with UC) are assessed. Blood is drawn and a stool sample is obtained. Patients undergo a sigmoidoscopy and six biopsies from the sigmoid region are taken.

\section{Visit 1 (T1)}

Visit 1 (T1) is performed 1 day after baseline. At T1, the patients receive a clinical examination, including an otolaryngological examination, and vital signs (heart rate and blood pressure), body temperature, medication, adverse events and HBI (patients with $\mathrm{CD}$ ) or partial Mayo Score (patients with ulcerative colitis) are assessed. 
Figure 3 Study design.

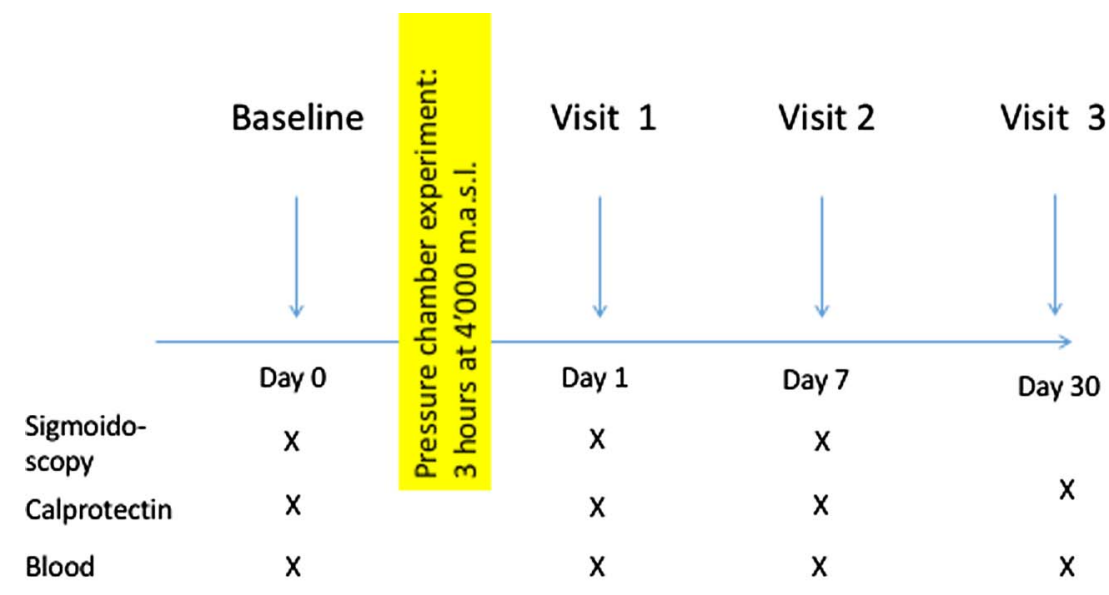

Blood is drawn directly before the stay in the hypobaric chamber. Patients then enter the hypobaric chamber (ascent within $10 \mathrm{~min}$, 3-hour exposure to hypoxic conditions simulating an altitude of 4000 m.a.s.l. (FL 13000 $\mathrm{ft}$,) afterwards controlled descent under continuous pulsoximetric control). During the 3-hour stay in the hypobaric chamber, repetitive measurements of the bladder volume are performed $(5 \mathrm{~min}$ after reaching the altitude of 4000 m.a.s.l., and at time points 30, 45, 60, 120, $180 \mathrm{~min}$ and after the descent) by ultrasonography. Directly after the stay in the hypobaric chamber, blood is drawn again. A sigmoidoscopy from the sigmoid region is performed (at the latest 2 hours after leaving the hypobaric chamber) and six biopsies, remote of previous biopsy areas, are collected.

\section{Visit 2 (T2)}

Visit 2 (T2) takes place 7 days after the stay in the hypobaric chamber. At T2, patients receive a clinical examination, and vital signs (heart rate and blood pressure), body temperature, medication, adverse events and HBI (patients with CD) or partial Mayo Score (patients with UC) are assessed. Blood is drawn and a stool sample is obtained, prior to a sigmoidoscopy where six biopsies from the sigmoid region, remote of previous biopsy areas, are taken.

\section{Visit 3 (T3)}

Visit 3 (T3) takes place 30 days after the stay in the hypobaric chamber. At T3, the patients receive a clinical examination, and vital signs (heart rate and blood pressure), body temperature, medication, adverse events and HBI (patients with CD) or partial Mayo Score (patients with UC) are assessed. Blood is also drawn and a stool sample is obtained.

\section{Study procedures}

\section{Sigmoidoscopy with biopsies from the sigmoid region}

To retrieve biopsies from the colon during the course of the study, three sigmoidoscopies are performed. For endoscopy, Olympus EVIS EXERA III (GIF-H190) and Olympus EVIS EXERA (CF-Q160) are used. The first sigmoidoscopy is performed 1 day before the stay in the hypobaric chamber. The second sigmoidoscopy is performed directly after the hypobaric chamber and the third sigmoidoscopy is performed 1 week after the hypobaric chamber. During each sigmoidoscopy, six biopsies are taken with standard size forceps $(2.4 \mathrm{~mm})$. One biopsy is analysed by real-time quantitative PCR for HIF-1, as well as pro-inflammatory and anti-inflammatory cytokines (eg, interferon- $\gamma$, IL-8, tumour necrosis factor, IL-1 $\beta$, IL-6, IL-10 and transforming growth factor- $\beta$ ), pattern recognition receptors (eg, toll-like receptor 4 and nucleotide-binding oligomerisation domain-containing protein (NOD) 2), inflammasome-associated proteins (NOD-like receptor family pyrin domain containing (NLRP) 3, IL-1 $\beta$, IL-18 and caspase 1), autophagy factors (eg, microtubule-associated proteins $1 \mathrm{~A} / 1 \mathrm{~B}$, light chain 3B (MAPLC3B) and sequestosome-1) and tissue remodelling factors (eg, matrix metalloproteinase 9 and smooth muscle $\alpha$-actin). One biopsy is analysed by western blot for HIF-1, nuclear factor (NF)- $\mathrm{B}$ signalling pathway activation (eg, phospho-NF- $\kappa \mathrm{B}$ and phospho-inhibitor of $\kappa \mathrm{B}$, autophagy factors (sequestosome-1, MAPLC3B and mammalian target of rapamycin) and inflammasomeassociated factors (eg, NLRP3). Another biopsy is analysed for gene expression by in situ hybridisation for pro-inflammatory and anti-inflammatory factors. Two biopsies are analysed by immunohistochemistry for phospho-NF- $\kappa \mathrm{B}$ and one biopsy is stained with $\mathrm{H} \& \mathrm{E}$.

\section{Faecal calprotectin}

Faecal calprotectin levels are measured at the University Hospital Zurich by a novel ELISA-based calprotectin test (EliA calprotectin, Thermo Scientific). ${ }^{23}$

\section{Measurement of the volume state}

The volume state was measured to evaluate possible high-altitude diuresis ${ }^{24}$ and to determine corresponding changes of volume-regulatory hormones.

\section{Measurement of bladder volume}

During the 3-hour stay in the hypobaric chamber, repetitive measurements of the bladder volume are performed 
(5 min after reaching the altitude of 4000 m.a.s.l., and at time points 30, 45, 60, 120, $180 \mathrm{~min}$ and after the descent). The volume of the bladder is assessed by ultrasonography.

\section{Variables in blood and urine}

Urine samples for the measurement of angiotensin and vasopressin are obtained before and after the hypobaric chamber. EDTA-blood is drawn before and after the hypobaric chamber for the measurement of renin, aldosterone, vasopressin and angiotensin.

\section{Sympathicotonic reaction}

Blood pressure and pulse are measured before and after the hypobaric chamber, as well as every $15 \mathrm{~min}$ during the stay in the hypobaric chamber. Furthermore, catecholamines (epinephrine and norepinephrine) are measured before and after the stay in the hypobaric chamber.

ANGII (Sigma-Aldrich, St. Louis, Missouri, USA) and aldosterone (R\&D Systems, Minneapolis, Minnesota, USA) concentrations are quantified by means of a competitive enzyme immunoassay and renin activity by a fluorometric assay kit (BioVision, Milpiates, California, USA).

\section{Assessment of safety}

Continuous pulsoximetric monitoring during the stay in the hypobaric chamber is performed. The minimum oxygen saturation, measured by pulsoximetric monitoring, that is accepted during stay in the hypobaric chamber is $70 \%$.

\section{Calculation of clinical activity}

To calculate the clinical activity of patients with $\mathrm{CD}$, the HBI is used. ${ }^{25}$ This index includes general well-being, abdominal pain, the number of bowel movements, abdominal resistance and CD-associated extraintestinal diseases. Each category has a point value assigned and from the sum of all categories a point value is calculated.

Patients with an overall value of $<5$ points are in clinical remission, patients with 5-7 points have mild, with 8-16 points moderate and with $>16$ points severe disease.

To calculate the clinical activity of patients with UC, the partial Mayo Score is used. ${ }^{26}$ This clinical index includes the stool frequency, the amount of blood in the stool and the physician rating of disease activity. Each category has a point value assigned from 0 to 3 and from the sum of all categories a point value was calculated. Remission is defined as $0-1$ points, mild disease 2-4 points, moderate disease 5-6 points and severe disease as $7-9$ points.

\section{Administration of patient records}

All documents containing patient data carry the respective patient code assigned by the physician. Encoded documents are stored at the Department of Gastroenterology and Hepatology, University of Zurich, Switzerland, and will be stored for 10 years. Only the study team has access to the patient codes. Data entry is performed continuously at the Department of Gastroenterology and Hepatology, and if data are missing, a research assistant investigates to obtain all information as required.

\section{Sample size calculation}

In a pilot study with healthy controls (Wojtal et al, unpublished), the ideal apical sodium-dependent bile acid transporter (ASBT) had a mean reduction of $49 \%$ compared with baseline measurements (SD 15\%). The power to detect a reduction of $67 \%$ among patients with IBD is then $80 \%$ with $\mathrm{n}=14$ patients and $\mathrm{n}=14$ controls. The calculation is based on a two-sided t-test of the $\log$ ASBT measurements with significance level of $5 \%$. About the same power is reached if $n=20$ patients are compared with $\mathrm{n}=10$ controls.

\section{Statistical data evaluation}

The statistical data evaluation will be performed by the Institute of Epidemiology, Biostatistics and Prevention at the University of Zurich, Switzerland. Statistical analysis of the clinical data will be performed using a paired Student's t-test, and probabilities ( $p$, two tailed) of $\mathrm{p}<0.05$ are considered statistically significant. For statistical analysis of groups, one-way analysis of variance (ANOVA) will be performed followed by the Tukey's Post Hoc test. Differences are considered significant at $\mathrm{p}<0.05$, highly significant at $\mathrm{p}<0.01$ and very highly significant at $\mathrm{p}<0.001$.

\section{Patient recruitment}

Twenty patients with IBD and 10 healthy controls are included in the study. The patients are recruited from the IBD clinic of the Division of Gastroenterology and Hepatology of the University Hospital Zurich. The healthy controls are recruited age-matched and sexmatched from colleagues of the study team.

\section{Patient informed consent}

Prior to study participation, patients receive written and oral information about the content and extent of the planned study. In case of acceptance, they sign the informed consent form. In case of study discontinuation, all material will be destroyed or the patient will be asked if he/she accepts that the existing material can be used for the study.

\section{Time frame of the study}

Recruitment of patients and healthy volunteers was started in May 2014. The first group of five healthy volunteers was included into the study on December 2014. The first five patients with IBD were included in March 2015, last data collection is ongoing and is planned to be completed until 31 December 2016. 


\section{DISSEMINATION}

The results will be published in a peer-reviewed journal and shared with the worldwide medical community.

\section{DISCUSSION}

The importance of environmental factors in the pathogenesis of IBD, including their disease-modifying potential, is increasingly recognised. One factor that has come to the fore is hypoxia, which seems to play an important role in inflammatory processes. ${ }^{3}$ Numerous investigations in animal models and humans suggest that hypoxia has a profound influence on the integrity and function of the gastrointestinal tract, epithelial barrier, immune system and microbial composition. Furthermore, an increasing body of evidence points to that exposure to hypoxia (as in airline travel and highaltitude journeys) could be associated with various gastrointestinal symptoms and may have an impact on the pathogenesis and course of disease in IBD. Furthermore, in murine models of IBD intracellular hydroxylases, a group of enzymes responsible for oxygen sensing and activation of adaptive transcriptional responses to hypoxia, were identified as a new class of therapeutic targets in IBD. ${ }^{27-29}$ However, the integrative mechanisms underlying the role of hypoxia in inflammation, and especially in the pathogenesis of IBD, are still not well defined.

Up to date, standardised conditions to evaluate the effects of hypoxia in IBD are lacking. Evaluating subjects at high altitudes, as it was done by our group with voluntary mountaineers, is complex, and is also mainly limited to healthy and athletic subjects, that are able to conduct such a journey. ${ }^{22}$ Furthermore, at high altitudes facilities to timely process blood and tissue are lacking and conditions for interventional diagnostics, like endoscopies, are limited. Therefore, we developed an environment where patients and healthy volunteers could be exposed to hypoxic conditions simulating an altitude of 4000 m.a.s.l. With this setup, we are able to perform prospective and controlled investigations under standardised conditions and still have access to the facilities of our University Hospital. Understanding clinical, molecular and microbiological consequences of intestinal hypoxia may ultimately derive further insights on the pathogenesis of IBD, far beyond exposure to lower partial oxygen pressure ambient air.

\section{Trial status}

The study procedures started in September 2015. Data collection was started in January 2016 and is ongoing.

\footnotetext{
Author affiliations

${ }^{1}$ Division of Gastroenterology and Hepatology, University Hospital Zurich, Zurich, Switzerland

${ }^{2}$ Division of Gastroenterology, Triemli Hospital, Zurich, Switzerland

${ }^{3}$ Zurich Center for Integrative Human Physiology (ZIHP), University of Zurich, Zurich, Switzerland

${ }^{4}$ Institute of Physiology, University of Zurich, Zurich, Switzerland
}

${ }^{5}$ Institute of Biostatistics, University of Zurich, Zurich, Switzerland

${ }^{6}$ Department of Intensive Care Medicine, Inselspital, Bern University Hospital, University of Bern, Bern, Switzerland

${ }^{7}$ Vetsuisse Faculty, Institute of Veterinary Physiology, Zurich, Switzerland

${ }^{8}$ Universidad Peruana Cayetano Heredia (UPCH), Lima, Peru

${ }^{9}$ Swiss Aeromedical Center, Dubendorf, Switzerland

${ }^{10}$ Institute of Experimental Immunology, University of Zurich, Zurich, Switzerland

${ }^{11}$ Institute of Pathology, University Hospital Zurich, Zurich, Switzerland

Contributors JZ, SV and GR were the initiators of this study. JZ wrote the study protocol. JZ, SV, SS, PAR, DB and LB conducted the study procedures. All other authors took part in data acquisition and study planning. JZ and SV wrote and revised the final manuscript and all authors read and approved it.

Funding This project is mainly supported by a grant from the IBDnet and by an unrestricted study grant from AbbVie (to GR, SV and JZ) and further supported by the NCCR Kidney.CH (to CL, RHW) and the ZIHP (to MG).

Competing interests None declared.

Ethics approval Ethics Committee of the Kanton Zurich, Switzerland (KEK-ZH-Nr. 2013-0284, 30 April 2014).

Provenance and peer review Not commissioned; externally peer reviewed.

Open Access This is an Open Access article distributed in accordance with the Creative Commons Attribution Non Commercial (CC BY-NC 4.0) license, which permits others to distribute, remix, adapt, build upon this work noncommercially, and license their derivative works on different terms, provided the original work is properly cited and the use is non-commercial. See: http:// creativecommons.org/licenses/by-nc/4.0/

\section{REFERENCES}

1. Vavricka SR, Rogler G, Biedermann L. High altitude journeys, flights and hypoxia: any role for disease flares in IBD patients? Dig Dis 2016;34:78-83.

2. Cummins EP, Keogh CE, Crean D, et al. The role of HIF in immunity and inflammation. Mol Aspects Med 2016;47-48:24-34.

3. Hartmann G, Tschop M, Fischer R, et al. High altitude increases circulating interleukin-6, interleukin-1 receptor antagonist and C-reactive protein. Cytokine 2000;12:246-52.

4. Vavricka SR, Rogler G, Maetzler S, et al. High altitude journeys and flights are associated with an increased risk of flares in inflammatory bowel disease patients. J Crohns Colitis 2014;8:191-9.

5. Eltzschig HK, Abdulla P, Hoffman E, et al. HIF-1-dependent repression of equilibrative nucleoside transporter (ENT) in hypoxia. $J$ Exp Med 2005;202:1493-505.

6. Eckle T, Faigle M, Grenz A, et al. A2B adenosine receptor dampens hypoxia-induced vascular leak. Blood 2008;111:2024-35.

7. Eltzschig HK, Ibla JC, Furuta GT, et al. Coordinated adenine nucleotide phosphohydrolysis and nucleoside signaling in posthypoxic endothelium: role of ectonucleotidases and adenosine A2B receptors. J Exp Med 2003;198:783-96.

8. Thompson LF, Eltzschig HK, Ibla JC, et al. Crucial role for ecto-5'-nucleotidase (CD73) in vascular leakage during hypoxia. $J$ Exp Med 2004;200:1395-405.

9. Rosenberger P, Schwab JM, Mirakaj V, et al. Hypoxia-inducible factor-dependent induction of netrin-1 dampens inflammation caused by hypoxia. Nat Immunol 2009;10:195-202.

10. Thornton RD, Lane P, Borghaei RC, et al. Interleukin 1 induces hypoxia-inducible factor 1 in human gingival and synovial fibroblasts. Biochem J 2000;350(Pt 1):307-12.

11. Vermeulen $\mathrm{N}$, Vermeire $\mathrm{S}$, Arijs I, et al. Seroreactivity against glycolytic enzymes in inflammatory bowel disease. Inflamm Bowel Dis 2011;17:557-64.

12. Karhausen J, Furuta GT, Tomaszewski JE, et al. Epithelial hypoxia-inducible factor-1 is protective in murine experimental colitis. J Clin Invest 2004;114:1098-106.

13. Giatromanolaki A, Sivridis E, Maltezos E, et al. Hypoxia inducible factor 1alpha and 2alpha overexpression in inflammatory bowel disease. J Clin Pathol 2003;56:209-13.

14. Shay JE, Imtiyaz HZ, Sivanand S, et al. Inhibition of hypoxia-inducible factors limits tumor progression in a mouse model of colorectal cancer. Carcinogenesis 2014;35:1067-77.

15. Tsuzuki $Y$, Fukumura $D$, Oosthuyse $B$, et al. Vascular endothelia growth factor (VEGF) modulation by targeting hypoxia-inducible 
factor-1alpha--> hypoxia response element--> VEGF cascade differentially regulates vascular response and growth rate in tumors. Cancer Res 2000;60:6248-52.

16. Aeberli I, Erb A, Spliethoff $K$, et al. Disturbed eating at high altitude: influence of food preferences, acute mountain sickness and satiation hormones. Eur J Nutr 2013;52:625-35.

17. Wojtal KA, Cee A, Lang S, et al. Downregulation of duodenal SLC transporters and activation of proinflammatory signaling constitute the early response to high altitude in humans. Am J Physiol Gastrointest Liver Physiol 2014;307:G673-88.

18. Siebenmann C, Bloch KE, Lundby C, et al. Dexamethasone improves maximal exercise capacity of individuals susceptible to high altitude pulmonary edema at $4559 \mathrm{~m}$. High Alt Med Biol 2011;12:169-77.

19. Gassmann M, Muckenthaler MU. Adaptation of iron requirement to hypoxic conditions at high altitude. J Appl Physiol 2015;119:1432-40.

20. Anand AC, Sashindran VK, Mohan L. Gastrointestinal problems at high altitude. Trop Gastroenterol 2006;27:147-53.

21. Wu TY, Ding SQ, Liu JL, et al. High-altitude gastrointestinal bleeding: an observation in Qinghai-Tibetan railroad construction workers on Mountain Tanggula. World J Gastroenterol 2007;13:774-80.
22. Fruehauf $\mathrm{H}$, Erb A, Maggiorini $\mathrm{M}$, et al. $\mathrm{T} 1080$ Unsedated transnasal esophago-gastroduodenoscopy at $4559 \mathrm{M}(14957 \mathrm{Ft})$-endoscopic findings in healthy mountaineers after rapid ascent to high altitude. Gastroenterology 2010;138:483-4.

23. http://www.phadia.com/PageFiles/29347/Product\%20information\% 20EliA\%20Calprotectin.pdf

24. Swenson ER. High altitude diuresis: fact or fancy. In: Houston CS, Coates G, eds. Hypoxia: women at altitude. Burlington, VT: Queen City Press, 1997:272-83.

25. Harvey RF, Bradshaw JM. A simple index of Crohn's-disease activity. Lancet 1980;1:514.

26. Lewis JD, Chuai S, Nessel L, et al. Use of the non-invasive components of the Mayo score to assess clinical response in ulcerative colitis. Inflamm Bowel Dis 2008;14:1660-6.

27. Cummins EP, Doherty GA, Taylor CT. Hydroxylases as therapeutic targets in inflammatory bowel disease. Lab Invest 2013;93:378-83.

28. Cummins EP, Seeballuck F, Keely SJ, et al. The hydroxylase inhibitor dimethyloxalylglycine is protective in a murine model of colitis. Gastroenterology 2008;134:156-65.

29. Tambuwala MM, Cummins EP, Lenihan CR, et al. Loss of prolyl hydroxylase-1 protects against colitis through reduced epithelial cell apoptosis and increased barrier function. Gastroenterology 2010;139:2093-101. 